\title{
A SECURITY EVALUATION CRITERIA FOR BASELINE SECURITY STANDARDS
}

\author{
W.J.Brooks ${ }^{1} \mathrm{M}$ Warren ${ }^{1}$ and W Hutchinson ${ }^{2}$ \\ ${ }^{I}$ Dept of Computing \& Mathematics, Deakin University, Geelong, Victoria, Australia.
}

${ }^{2}$ School of Computing \& Information Science, Edith Cowan Universit, Mount Lawley, Western Australia, Australia.

Key words: Evaluation Criteria, Risk Analysis and Baseline Security.

\begin{abstract}
Computer security is now recognised as an important consideration in modern business, with a variety of guidelines and standards currently available to enable different business environments to be properly protected. However, financial and operational constraints often exist which influence the practicality of these recommendations. New baseline security methods such as Australian and New Zealand Standard (AS/NZS) 4444 and British Standard (BS) 7799 represent minimal standards which organisations can use to improve their security. The aim of the paper is to look at the effectiveness of baseline security standards through the use of an evaluation criteria, which assesses their effectiveness.
\end{abstract}

\section{INTRODUCTION}

During the last few decades the use of Information Technology has become more widespread in all areas of society, and the types of activities that it performs or supports have become increasingly more important. As a result, information systems are now heavily utilised by all organisations and relied upon to the extent that it would be impossible to manage without them. The growth of organisational business internetworking via e-commerce 
applications present further opportunities for unauthorised access to information systems (Lyre, 1999) and this is a now a major worry. Can risk analysis help to protect organisations? The aim of risk analysis is to eliminate or reduce risks and vulnerabilities that affect the overall operation of organizational computer systems. Risk analysis not only looks at hardware and software, but also covers other areas such as physical security, human security, and business and disaster protection. In practice, there are major problems with the use of risk analysis; the time taken to carry out a review, the cost of hiring consultants and/or training staff. To overcome these negative aspects, baseline security standards were developed. Baseline security standards offer an alternative to conventional risk analysis methods as they represent the minimally acceptable security countermeasures that an organization should have implemented. These countermeasures are applied in a generic manner, e.g. every organization should have the same baseline security countermeasures.

The advantages of using baseline methods include (Warren, 1997):

- it is cheap to use;

- it is simple to use;

- no training is required to use the method;

- it is quicker then undertaking a full security review.

The disadvantages of using baseline methods include (Warren, 1997):

- the generic nature of baseline security methods mean they may not solve all of the organizational security requirements;

- the fact that they have been designed for use within a general environment mean that they may not be suited for all environments, i.e. healthcare or small businesses;

- there is no suggestion about how the security countermeasures may be implemented;

- they do not contain cost benefit details.

A number of baseline security methods have been developed in recent years. But do these baseline security standards actually offer a minimal level of protection, can organisations expect the correct advice to be offered by these baseline security standards? This was the rationale behind developing an evaluation criteria that could be used to demonstrate how effective baseline security methods are. 


\section{RESEARCH BACKGROUND}

This section describes the steps involved in the development process of the evaluation criteria and the baseline security evaluation framework.

\subsection{Analysis of Information Security Threats, Controls, and Baseline Standards}

A content analysis was performed on existing information security threats (i.e. viruses), controls (i.e. anti-virus software), and information security baseline standards to determine a set of generic components that related to malicious software. A content analysis is research method that is used to place the raw data collected into logical, meaningful categories (Strauss \& Corbin, 1990).

\subsection{Development of the Evaluation Criteria for Malicious Software}

Stage 2 was concerned with transforming the research generated from stage 1 into an evaluation criteria. The evaluation criteria contains four categories each listing the definitions of each component based on the content analysis of information security. These are:

-The Information Security category relates to the information security management system (ISMS), which is concerned with policies, regulations and rules that IT management would establish and implement.

-The Origin of Threats category relates to the types of threats, vulnerabilities and attacks that the system could be at risk to.

-The Security Controls category relates to the generic security controls, countermeasures and safeguards that an organisation can implement to reduce the risks to the system.

- The Recover and Containment category relates to procedures, policies and regulations that should in place to help an organisation recover from a disaster or be ready for the unexpected. This category also covers procedures on information backup.

The evaluation criteria for malicious software is shown in Table 1

Table 1. The Evaluation Criteria Used

1. Information Security

a. ISMS Level

2. Origin of Threats

a. Organisational 

b. Human Failure
c. Deliberate Acts

3. Security Control
a. Security Awareness
b. Authorisation Security
c. Virus Protection
d. Data Integrity
e. Web Security
f. Encryption Technologies
g. Organisational Security
h. Hardware and Software Security

4. Recovery and Containment
a. Disaster Recovery
b. Contingency Planning
c. Information Backup

\subsection{Analysis of Existing Baseline Security Frameworks}

The criteria was applied against a variety of security baseline standard, which were.

- Australian Communications-Electronic Security Instruction 33 (ACSI 33) (ACSI, 2000);

- Australian / New Zealand Standard for Information Security Management (AS/NZS 4444) (Standards Australia, 1999, Standards Australia, 2000);

- German IT Baseline Protection Manual (BSI, 2000).

A full discussion of the baseline methods is found later in the paper.

\subsection{Evaluation of Results}

The results are evaluated and analysed using a evaluation framework that was based on the research undertaken by Van de Haar and Von Solms (Van de Haar \& Von Solms, 1993). Within their research they developed an evaluation criteria that could be applied to generic security risk analysis. They determined that Information Security Management (ISM) contains five operational security environments (OSEs) (Von Solms et al., 1992). These five OSEs are as follows:

- The Ideal OSE: an environment as described in the information security policy. This level is not always obtainable; 
- The Prescribed OSE: an environment as dictated by external parties;

- The Baseline OSE: an environment that has been set as a goal by top management and is reachable by the organisation;

- The Current OSE: the current state of security within the organisation; and,

- The Survival OSE: the minimum set of information security controls and counter-measures that are needed to stay operational.

Within our research we were looking at baseline security risk analysis and determined that the baseline security evaluation criteria would be based on three of the OSEs: These are:

-Ideal OSE (High rating) and is the highest attainable level of security that can be implemented by an organisation.

- Baseline OSE (Medium rating) and is the minimum level of security that should be implemented by an organisation for their system to be secure.

- Survival OSE (Low rating) and is the minimum level of security measures needed to for an organisation to stay operational, but the system is classified as insecure.

This aspect of our research is particularly important because it describes the initial development of the new evaluation framework for baseline security risk analysis. Replacing the concept that baseline security is just a single level of security, but showing that baseline security could actually be represented by a number of different levels. As we stated before the limitations of our approach, is that we are just looking at the malicious aspects of the security baseline standards. Table 2 , shows the structure of the criteria evaluation, and the rating that would be used based upon the content analysis, which was described earlier.

Table 2. Example of Evaluation Criteria

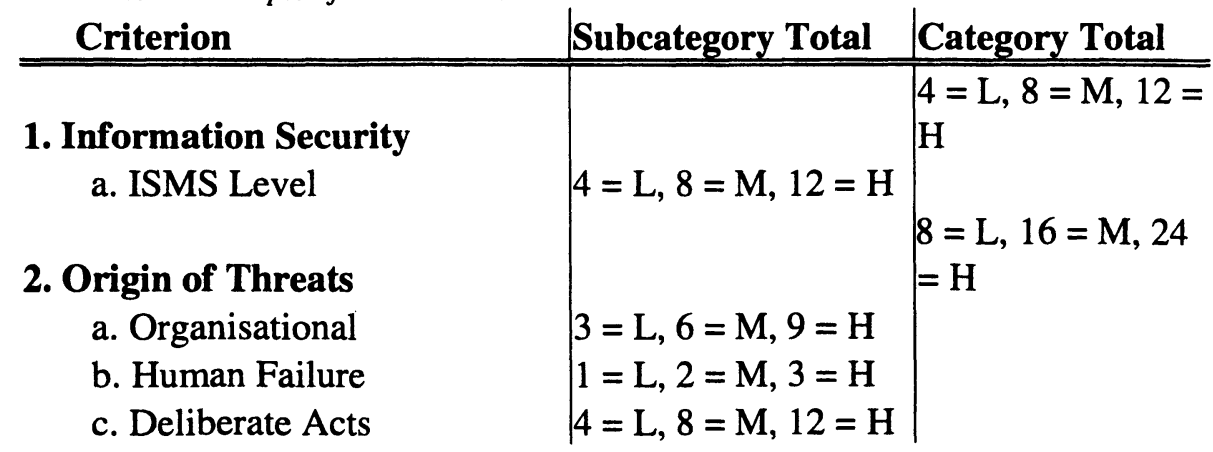




\section{Security Controls}

a. Security Awareness

b. Authorisation Security

c. Virus Protection

d. Data Integrity

e. Web Security

f. Encryption Technologies

g. Organisational Security

h. Hardware and Software Security
4. Recovery and containment

a. Disaster recovery

b. Contingency planning

c. Information Backup

$$
\begin{array}{|l|l} 
& \mid 31=\mathrm{L}, 62=\mathrm{M}, \\
5=\mathrm{L}, 10=\mathrm{M}, 15= & \\
\mathrm{H} & \\
3=\mathrm{L}, 6=\mathrm{H}, 9=\mathrm{H} \\
3=\mathrm{L}, 6=\mathrm{M}, 9=\mathrm{H} \\
6=\mathrm{L}, 12=\mathrm{M}, 18= \\
\mathrm{H} \\
3=\mathrm{L}, 6=\mathrm{M}, 9=\mathrm{H} \\
4=\mathrm{L}, 8=\mathrm{M}, 12=\mathrm{H} \\
4=\mathrm{L}, 8=\mathrm{M}, 12=\mathrm{H} \\
3=\mathrm{L}, 6=\mathrm{M}, 9=\mathrm{H} \\
\\
1=\mathrm{L}, 2=\mathrm{M}, 3=\mathrm{H} \\
1=\mathrm{L}, 2=\mathrm{M}, 3=\mathrm{H} \\
1=\mathrm{L}, 2=\mathrm{M}, 3=\mathrm{H}
\end{array} \mid \begin{aligned}
& \\
& 3=\mathrm{L}, 6=\mathrm{M}, 9=
\end{aligned}
$$

In table 2 shows the examples structure of the breakdown of evaluation criteria results in the following way:

- Individual results represents the value for each component (i.e. assets to be protected);

- Subcategory total represents the value that was obtained by adding together the values of each component within that subcategory; and,

- Category total represents the value that was obtained by adding together the values of each subcategory within that category.

\section{BASELINE EVALUATION RESULTS}

As mentioned before three security standards were assessed, these were:

Australian Communications-Electronic Security Instruction 33 (ACSI 33) The ACSI 33 (Australian Communications-Electronic Security Instruction 33) is a government standard developed by the Defence Signals Directorate (DSD) to provide guidance to Australian Government agencies on how they could protect their information systems (ACSI, 2000). These information systems may hold classified or non-classified information, but all should have some degree of protection if a reliable or accurate service is to be maintained. The ACSI 33 is intended for IT specialists only, such as, for example, IT security administrators. The standard is made up of a series of 
handbooks each covering a specific topic (i.e. Risk Management) with relevant security issues and, where appropriate, endeavours to categorise the security countermeasures into identified risk levels, defined as "grades"(ACSI, 2000). These grades have been formulated to provide a minimum policy level that should be implemented. The ACSI 33 has been written to be consistent with the Commonwealth Protective Security Manual (PSCC, 2001), and with two Australian Standards, AS/NZS 4444:1999 and AS/NZS 4360:1999 (ACSI, 20 00).

\section{Australian / New Zealand Standard for Information Security Management (AS/NZS 4444)}

The AS/NZS 4444:1999 is intended for use by managers and employees who are responsible for initiating, implementing and maintaining information security within their organisation and is considered a basis for developing organisational security standards (Standards Australia, 1999). It is an internationally recognised ISO standard (ISO/IEC 17799) (ISO/IEC, 2001). The standard has two parts: AS/NZS 4444.1:1999 (Standards Australia, 1999) and AS/NZS 4444.2:2000 (Standards Australia, 2000).

Part 1, "Code of Practice for Information Security Management", is identical to the British Standard (BS) 7799.1:2000 (British Standards, 2000a). It takes into account recent developments in information processing technology, and changes the emphasis towards information security to encompass information and IT projects, resources, networks and projects (Standards Australia, 1999). A comprehensive set of controls comprising the best information security practices currently in use are provided in this standard, which means that organisations are able to pick the guidelines most appropriate to them. The guidance and recommendations provided throughout this standard can be used as a basis from which, for example, a corporate policy can be developed, and should not be quoted as if they were specifications (Standards Australia, 1999).

Part 2, "Specification for Information Security Management Systems", is identical to the BS 7799.2:2000 (British Standards, 2000b) and forms the basis for an assessment of the ISMS of an organisation, and can be used as a basis for a formal certification scheme (Standards Australia, 2000b). It specifies requirements for security controls to be implemented according to the needs of individual organisations.

\section{German IT Baseline Protection Manual}

The IT Baseline Protection Manual is a nationally recognised standard in Europe developed by the German Federal Agency for Security in Information Technology (BSI) and presents a detailed set of standard 
security measures that apply to virtually every IT system (BSI, 2000). The aim of these security recommendations is to achieve a security level for IT systems that will satisfy normal protection requirements and can also serve a basis for other systems and applications requiring a higher degree of protection. This is achieved through appropriate application of organisational, personnel, infrastructural and technical standard security safeguards (Henze, 2000). The IT Baseline Protection Manual includes (Henze, 2000):

- Standard security measures for typical IT systems with "normal" protection requirements;

- A description of the threat scenario that is globally assumed;

- Detailed descriptions of safeguards to assist with their implementation;

- A description of the process involved in attaining and maintaining an appropriate level of IT security; and,

A simple procedure for ascertaining the level of IT security is attained in the form of a target versus actual comparison. Each standard was evaluated using the criteria described previously, with each of the four key criteria components be assessed. The criteria evaluation generated twelve sets of results (based upon the structure shown by table 1). We have summarised the results in Figure 1 in order to show a comparative representation of the results of each standard.

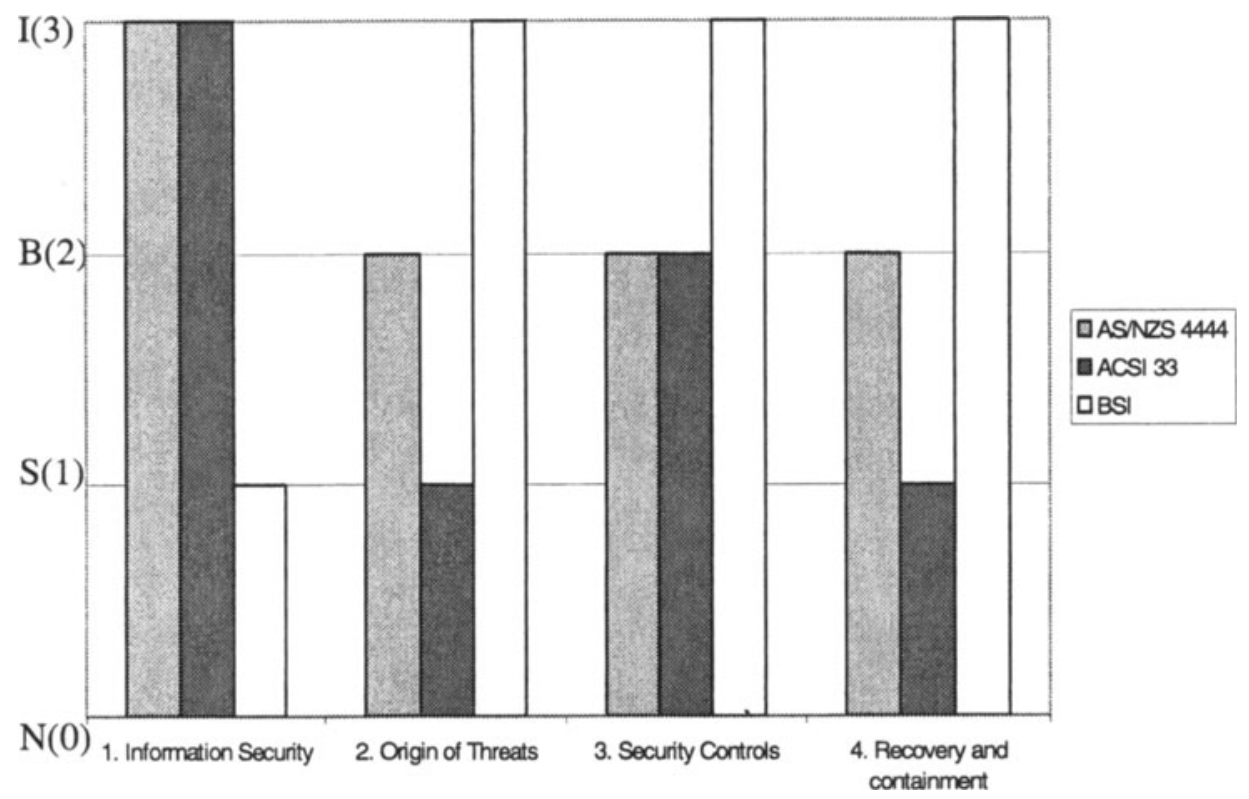

Figure 1. Information Security Baseline Standards Grouped 
From the analysis of the results obtained by the criteria evaluation on malicious software, the following conclusions have were made:

1. The ACSI 33 is a standard for the management of security controls and its main focus is providing appropriate security controls for web security. But it lacks detail on threats and recovery and containment procedures, which is a major issue for government agencies that require a high level of security due to the sensitive nature of data that is stored in their systems;

2. The AS/NZS 4444 is a standard for the management of security controls and its main focus is security awareness and authorisation security. Even though it provides more information on threats and recovery and containment procedures then the ACSI 33, it still does not provide sufficient technical details on security controls to allow organisations to maintain a secure system (i.e. it says what should be there to protect the organisation, but does not mention how to implement it); and,

3. The BSI is a standard for the technical implementation of security controls and it focuses on deliberate acts, web security, hardware and software security, and recovery and containment procedures. However, the standard lacks in detail on the steps involved in developing IT management security policies, which can be a problem for organisations that require guidance on writing security policies because they will not be aware of what is required in a security policy, thus incorrect procedures could be implemented resulting in a system being insecure.

\section{IMPACT UPON BASELINE SECURITY STANDARDS}

The evaluation of the baseline risk analysis security standards have shown that there is a distinct difference between the different standards. Even though it would be assumed that all baseline standards would be of the same standing. In terms of what the evaluation means for the each of the standards, in reality very little. Each standard was designed for a particular target audience and as such fulfils the target audience requirements. But as stated before, it is no longer the case that baseline security standards should be assumed to be of an equal level of standing in terms of protection. 


\section{CONCLUSION}

Information security baseline standards have been developed to provide security safeguards, implementation advice and aids for numerous IT systems in use today. The three standards looked at in this study, the AS/NZS 4444:1999 standard (Standards Australia, 1999, Standards Australia, 2000) the BSI standard (BSI, 2000), and the ACSI 33 standard (ACSI, 2000) each suggests a 'safe' minimal level of security that organisations can implement. The problem is that many IT professionals view baseline security differently and this can cause confusion for organisations, as they cannot always make a well-informed decision on which standard best suits their needs.

Assessment of the three baseline standards by use of the security evaluation criteria illustrate that not all baseline standards provide the same level of information on security controls and countermeasures. The new baseline security evaluation framework shows that baseline security is not just one level but three individual levels (ideal, baseline and survival). The new criteria based upon malicious software was assessed against three baseline standards. The results illustrate that the three standards provide different levels of information for ISMS, threats, security controls, and recovery and containment procedures, even though all three are considered to be baseline standards and of the same level.

We have illustrated within this paper that baseline security risk analysis methods are not all equal.

\section{REFERENCES}

ACSI (Australian Communications-Electronic Security Instruction) (2000) Instruction 33,

Defence Signals Directorate, Australia.

Available from: http://www.dsd.gov.au/infosec/acsi33/acsi index.html

British Standards (2000a) "BS7799: 1 Information Technology: Code of practice for information security management", British Standards, London, UK.

British Standards (2000b) “BS7799: 2 Information Security Management: Specification for information security management system", British Standards, London, UK.

BSI (2000) "IT Baseline Protection Manual", Federal Agency for Security in Information Technology, Germany. Available from: http://www.bsi.de/english/ 
Henze D (2000). “IT-Baseline Protection Manual”, BSI, Federal Agency for Security in Information Technology, Germany.

ISO/IEC (2001), "ISO/IEC 17799Information Technology - Code of practice for information security management", $1^{\text {st }}$ Ed., International Organization for Standardization, September.

Lyre, R. (1999) Information Security Management - Certification Scheme, Volume 20, No 2, The Australian Standard, Australia.

PSCC (Protective Security Coordination Centre) (2001) "Commonwealth Protective Security Manual", Attorney General's Department, Australia. Available from: http://www.ag.gov.au

Standards Australia (1999) AN/NZS 4444 "Information Security Management, Part 1: Code of practice for information security management", Standards Australia International Ltd \& Standards New Zealand.

Standards Australia (2001) AN/NZS 4444 Standards Australia/Standards New Zealand, "Information Security Management, Part 2: Specification for information security management systems", Standards Australia International Ltd \& Standards New Zealand.

Strauss A. \& Corbin J. (1990), "Basics of qualitative research: Grounded theory procedures and techniques", Newbury Park, Sage Publications Inc., California.

Van de Haar H. \& Von Solms R. (1993), “A Tool for Information Security Management”, Information Management \& Computer Security, Vol. 1 No. 1, pp. 4-10, MCB University Press Ltd, UK.

Von Solms et al. (1992) Von Solms R., Von Solms S.H. \& Caelli W.J., “An Information Security Management Model”, Proceedings IFIP WG 11.1 Workshop, , Singapore.

Warren M.J. (1997) "A new hybrid approach for Risk Analysis", IFIP WG11.1 Information Security Management Conference, Copenhagen, Denmark, pp. 123 - 130. 